This document is the accepted manuscript version of the following article: Muster, M., Hameed, A., Wood, D., Appleby-Thomas, G., \& Wasmer, K. (2019). Damping of post-impact vibrations. Applied Acoustics, 156, 427-433. \$ https://doi .org/10.1016/j.apacoust.2019.07.040

This manuscript version is made available under the CC-BY-NC-ND 4.0

license http://creativecommons.org/1icenses/by-nc-nd/4.0/

\title{
1 Damping of Post-Impact Vibrations
}

\section{Michael Muster, first author}

Centre for Defence Engineering, Cranfield University, Defence Academy of the United Kingdom

Shrivenham, SN6 8LA, UK

michael.muster@cranfield.ac.uk

\section{Amer Hameed, second author}

Centre for Defence Engineering, Cranfield University, Defence Academy of the United Kingdom

Shrivenham, SN6 8LA, UK

a.hameed@cranfield.ac.uk

\section{David Wood, third author}

Centre for Defence Engineering, Cranfield University, Defence Academy of the United Kingdom

Shrivenham, SN6 8LA, UK

d.wood@cranfield.ac.uk 


\section{Gareth Appleby-Thomas, fourth author}

Centre for Defence Engineering, Cranfield University, Defence Academy of the United Kingdom

Shrivenham, SN6 8LA, UK

g.thomas@cranfield.ac.uk

\section{Kilian Wasmer, fifth author}

Laboratory for Advanced Materials Processing, EMPA, Swiss Federal Laboratories for Materials Science And Technology

3602 Thun, Switzerland, $\mathrm{CH}$

kilian.wasmer@empa.ch

Keywords: flexural vibrations, damping, metallic plate, boundary reflection 


\section{Abstract}

Impacts of solid bodies on rigid plates produce loud noise and strong vibrations. During this impact, flexural waves travel circularly outwards from the point of impact. These waves are used to determine the properties of the impacting body. For accurate location and momentum measurements, it is necessary that the set of flexural waves pass acceleration sensors just once without being disturbed by reflections.

Different plate designs are tested with the aim to evaluate the shape, which offers the best damping properties against strong single impact.

The investigations showed that the damping properties of the plates are significantly better with a star-shaped design, which is equipped with a damping layer. The novelty presented here is that the damping properties are significantly increased using a star-shaped plate with specially power law formed and damped edges.

The work offers a solution to get significant better damping properties and a better signal for impact investigations. The results demonstrate that it is a promising approach for an impact detection system, which could be equally applicable to acoustic damping applications.

\subsection{Introduction}

This study investigates the effect of different plate shapes on their damping behaviour after a single excitation burst. The focus is on a short-time scale from microseconds up to milliseconds. Small-arms projectiles are structures that weigh from 2 to 10grams, which travel at supersonic speed and produce strong acceleration and acoustic signals when they impact. Due to their highspeed and rotation rates, they are generally difficult to characterise during flight and impact. The impact characterisation is used for applications like the estimation of rangedanger areas after deflection of the projectile on oblique plates. For such purposes one needs to know the position of impact and the momentum transmitted into the plate. With this information, one can estimate the residual energy and deflection angle of the projectile after impact. The main challenge of the 
impact detection is connected to huge decelerations and large deformations in a short time regime. Such projectiles are often too small to be equipped with sensors. Therefore, sensors mounted on witness plates are widelyused for impact detection devices. So-called Impact Soft-Recovery Experiments[1]are made, for example, with impact detection devices. In this case, the target under investigation is a brittle plate, monitored by an interferometer. The projectile impact generates strong vibrations, the characterisation of which helps to calculate the location and nature of the impact. Espinosa et al.[2] showed that it is possible to get a cleaner raw signal from the target plate by using a star-shaped flyer geometry. Their tests revealed that theeffect of the outer layer of the plate on the impact zone itself could be minimised significantly. Besides interferometers, accelerometers are frequently used to determine properties of the impact likethe impact position or the transferred momentum[3]-[5]. One ofthe major sources for measurement inaccuracies are random andreflected vibrations[6]. Hammetter et al. used an array of accelerometers fixed on the plate to determine the momentum transferred. They showed that geometrical properties of thedetecting plate itself might lead to inaccurate measurements. Another source of measurement inaccuracy is electronic filtering of the acceleration data. Best practice for severe-shock investigations is to use mechanically insulated accelerometers[7]. Severe mechanical shocks such as caused by bullet impact, typically lead to six degrees of freedom accelerations represented in broadband frequencies. This makes it difficult to determine the overall momentum [6], or the position. Mechanical insulators combined with electrical filters were found to be an appropriate way to over-come this problem. The impacting body excites the witness plate within a very short time in a nonlinear and random vibration regime, where scattering and reflections of vibrations at boundaries will occur[8], [9]. Right after the impact is the moment when the point of interest occurs. This moment is called the Arrival Time (AT). The AT is defined as the time when the sensor detects the first set of waves, which originate from the impact position. Consequently, knowing the exact AT is necessary to recalculate the exact impact position[10]with the Time Difference of Arrival (TDOA)algorithms. TDOA algorithms are often used nowadays, and optimised 
for passive tracking of wireless communication systems [11]. The underlying computations for wireless-devices tracking and impact location, however, are the same. The main issue for an accurate triangulation of impact remains the accurate AT detection. For flexural group waves travelling in steel at a speed of more than $2500 \mathrm{~m} / \mathrm{s}$, even minor errors lead to large positioning errors. The speed of sound in the target material and the AT difference must be known for positioning. Furthermore, by knowing the sensors positions, one can easily calculate the position of the impact/of the origin of the waves by numerical methods [12]. Mingzhouet al. showed the possibility of precisely detecting the AT of flexural waves using accelerometers after the impact of a test weight dropped on a large steel plate, even in noisy environments like power plants [13]. They used a sophisticated decomposition algorithm combined with the Hilbert Huang Transformation. They found that the proposed algorithm was capable of detecting the AT with a precision of several milliseconds. The main reason for the inaccuracies was still the noise in the signal. During a ballistic impact on a plate, stochastic waves appear inevitably, which affects the precision of the AT calculation. In an idealised case, flexural and compression waves emitted from the origin of impact would not be reflected. This can be realised by two approaches: Using a plate significantly larger in comparison with the investigated area (this approach is presented in [9]), or using a special damping plate. A very interesting approach is a plate of special shape which decreases in thickness at the edge (called a wedge shape) in a power-law profile [14]. The different waveforms are eliminated due to internal refraction. However, the power-law shape is difficult to manufacture, which is why it is not used in practical vibration dampers [15]. Possible ways of manufacturing this wedge shape are 3D milling or casting [16], both of which are expensive. For hardened-steel and supersonic impacts, such delicate and large structures are normally unsuitable to be made by industrial methods. There are some highly effective damping alternatives which are easier to manufacture, i.e. the application of a thin absorbing layer to the plate surface [14]. Another possibility is an acoustic black hole, reduced in its space requirements as proposed by [17]. This showed that a damping layer covering and wrapping the acoustic black hole results in a reduced vibration. 
However, for impact analysis purposes, other shapes are more desirable. From a manufacturing point of view, if one wants to pro-cess a hardened wear plate, just 2D shapes should be used. Such plate designs can be produced by waterjet or plasma cutting. Possible shapes that can be produced easily are polygons or any kind of round, but two-dimensional. Star-shaped (polygonal) flyer plates are also able to trap compression waves, as used for normal impact soft-recovery experiments [2]. With the star-shaped plate, much lower level reflection is observed during impact. This enhances the quality of the raw data in ballistic experiments. The edge morphology can serve as a trap for waves and was investigated computationally in [18]. Apart from the computational investigations, the real noise-damping properties of the plates need to be quantified. The plate experiences random vibrations after significant impulse is applied. The plate's response cannot be assessed using purely analytical models [8]. The randomdecrement method is a possible way to quantify the damping factor of a system. This approach is suitable for systems which are randomly excited, for example fluid flow against rigid structures like ships or bridges [19]. Using the logarithmic decrement to determine the damping ratio is widely used for such damped systems [20]. However, this system is unsuitable for the present application because the plate is just excited once with a strong burst, then recovers fully as the vibration decays down to non-excited state. The random decrement method would be more applicable if the system was excited by many fragments impacting simultaneously. In the case of a strong excitation burst, the plate vibrates stochastically. According to the experimental results of Humbertet al.[8], all frequencies are abundant during the first $70 \mathrm{~ms}$ after excitation with one strong mechanical burst. All frequency bands of the broadband spectra of the free vibrational decay have different decay rates. Itis not possible to calculate just one valid damping ratio [21], especially if one wants to calculate the AT, which is the case in this investigation. In this case, one can use band-pass filters to determine each specific damping ratio. The drawback of this system is that the results are strongly dependent on the chosen band-width of the band-pass filter [22]. The information about the properties of the impacting mass and the AT is retained in flexural waves only for the first few post-impact milliseconds. To get a meaningful 
result, it is desirable that the first $100 \mathrm{~ms}$ are not disturbed by reflections. It is possible to sort out specific reflections using wave separation [23]. This approach, however, works for ultrasonic signals because small changes in frequency may be detected with such systems. Severe shock signals need to be detected with mechanically prefiltered accelerometers. The recorded acceleration broadband signal decays exponentially after a single excitation event. This is comparable to the decay of sound in room acoustics. In room acoustics, the "reverberation time" is a common way of describing a signal decay pattern. The reverberation time is defined as an exponential decay fit, which has the advantage that there is just one main decay rate, which includes all frequencies. This reverberation time was introduced by Schroeder [24] in 1965. He used filtered pistol shots to produce a single excitation event comparable to the application described in this paper. Since the damping is directly related to the reverberation time [25], this is a meaningful quantity for the system performance in the present case. The reverberation time is mostly measured to determine room acoustics [26], but can also be measured for any solid plates, for instance in musical equipment [27]. This paper describes different plate shapes and their capabilities to attenuate the noise, relative to a reference plate. The increased damping properties enhance the detection of the AT. The vibrations are detected with accelerometers capable of measuring uniaxial accelerations. They are normally positioned on the plate. This means that only flexural waves will be measured. Because of the similarities to the acoustical reverberation of a sound burst (or filtered pistol shot), the reverberation time is chosen as quantification method. In an initial study, a drop test is performed in a small-scale experiment to determine the most efficient plate shape. This shape is produced in a larger scale model and used for ballistic impact tests.

\subsection{Experimental Procedures and Materials Tested}

\subsubsection{Test Procedures}

Two different test scenarios were chosen to estimate the scalability of the system. The goal of the two scenarios is to evaluate and assess the damping properties of plate shapes after impact. The most promising design after the first test 
scenario is verified on a larger scale. All shapes were produced using the waterjet cutting technique which results in a surface roughness of $\mathrm{Ra} 25 \mu \mathrm{m}$. This is comparable to standard sandblasting. The overall tolerances is $0.1 \mathrm{~mm}$, independent of the plate thickness. Concave edges are produced with radii of $0.75 \mathrm{~mm}$, convex edges with minimum radii of $0.08 \mathrm{~mm}$. In the case of the shapes subject to the power law, the exponent was approx. 2.8 for both test scenarios. The surface of the plate has a Ra of $8 \mu \mathrm{m}$, which is relatively smooth. The surface of the plate has no disturbances except for the screwed-in accelerometers. The data acquisition system for the accelerometers was the NI USB-6366 (National Instruments, USA) in both scenarios, with simultaneous sampling, acquiring and recording data every $0.5 \mu \mathrm{s}$. Simultaneous sampling is very important for such tests, so that the exact time shift between the excitation events is measured. The acceleration sensors used for both scenarios are shock sensors 350B01 (PCB, USA), mechanically isolated and electrically filtered. The signal conditioner was the $483 \mathrm{C} 40$ (PCB, USA).

The data acquisition time was set to $150 \mathrm{~ms}$, i.e. long enough to investigate the signal decay. Each test was repeated three times. The maximum signal amplitude acquired was normalised to \pm 1 . With this approach, one can compare lowmomentum impacts with high-momentum impacts. The time from primary wave detection until $1 \%$ of the max amplitude is reached, was used to calculate the reverberation time of the different plates. This was necessary to quantify and compare the results.

\subsubsection{First Test Scenario}

The first test scenario was a small-scale setup; plates of four different shapes were used to acquire information about their damping behaviour. All plates for the first set of experiments consisted of $5 \mathrm{~mm}$ thick S275J2 steel. As a reference, a simple rectangular plate was analysed. This had four additional small rectangles attached for fixation purposes as shown in Figure 2-1. Most ballistic standard tests rely on square or rectangular plate shapes. Square plates are used in the interest of simplicity and cost savings. A second reference plate of oval shape was used to examine whether this would enhance the damping properties. The 
third plate was made with projections featuring a $60^{\circ}$ angle in accordance to the findings of [2], [18], called the "star-shaped with $60^{\circ}$ edge" plate. The height of the triangle is $35 \mathrm{~mm}$. It is assumed that, with this shape, the damping rate increases significantly. The fourth shape is the combination of the power-law profile and the star-shape called "star-shape with power-law edge", the edges are shown in Fig. 2c. The number of spikes of the fourth shape is the same as in the case of the star-shaped plate. The width between the corners remained the same. However, the length of the edge increased to $98 \mathrm{~mm}$. As suggested in [14], the surface was treated with an absorbing surface, a magnetic polymer compound. This layer can be removed if required. The star-shaped with $60^{\circ}$ edge plate and the star-shaped plate with power-law edges were equipped with three thread bores each to accommodate three sensors. One of these bores is closer to the centre, while the second is near the concave edge of the spike and the third in the centre of the convex edge as shown in Figure 2-2. In this way, reflections and backscatter can be detected by monitoring and comparing the sensor data. However, sensor position and the speed of sound in a given material are parameters which can be controlled very accurately.

The plates were impacted by a falling steel roller bearing ball, its layout shown in Figure 2-2. The ball's mass was $16.84 \mathrm{~g}$, representing a widely used $16 \mathrm{~mm}$ ball bearing. The reason for using such an object was that it consists of $100 \mathrm{cr} 6$ hardened steel. These drop weights can sustain several drops without any deformation. The release height was $3.8 \mathrm{~m}$, the release mechanism an electromagnet switched by a transistor. The standard deviation of the overall drop accuracy was $0.1 \mathrm{~mm}$. This drop height delivered a kinetic energy of 0.62 Joule.

The damping layer was applied only to the last section of the power-law spikes. The size of the damping layer material $(10 \mathrm{~mm} \times 20 \mathrm{~mm} \times 1 \mathrm{~mm})$ was the same in all analysed cases as shown in Figure 2-2. The damping layer was placed on the oval plate, the star-shaped $60^{\circ}$ edge plate and the star-shaped plate with powerlaw edges. 
For the plates with geometries such as the ones in Figure 2-1, it was possible to add an equally distributed magnetic damping layer on the spikes or attach an equally distributed magnetic damping layer to the oval plate. This is not possible in the case of the rectangular plate. The reason is that the rectangular geometry has less edges and they are not equidistant.

\subsubsection{Second Test Scenario}

The second experiment was made using a $12 \mathrm{~mm}$ thick Hardox 500 tempered steel plate exhibiting a yield strength of $1300 \mathrm{MPa}$ [28] as target, against a supersonic impacting projectile.

Two different plates were tested in this scenario: A rectangular reference plate (Figure 2-1a) and a star-shaped plate with power-law edge (Figure 2-1d). Both plates had a thickness of $12 \mathrm{~mm}$, the reference plate was $700 \mathrm{~mm}$ wide and $800 \mathrm{~mm}$ long, while the star-shaped plate with power-law edge (Figure 2-1d) had a diameter of approx. $800 \mathrm{~mm}$. The reference plate was fixed in a rigid frame at the four corners as shown in the Figure 2-3. The power-law edged plate was mounted between a frame consisting of two steel rings and then fixed into the rigid frame shown in Figure 2-3a. A rubber inlay (called rubber damper) was mounted between the spikes and served for both damping and fixation purposes.

(a)

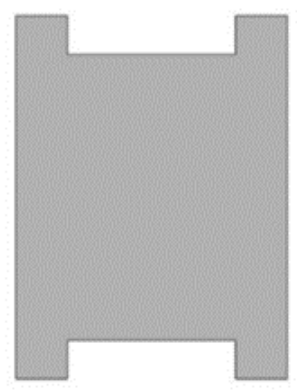

(b)

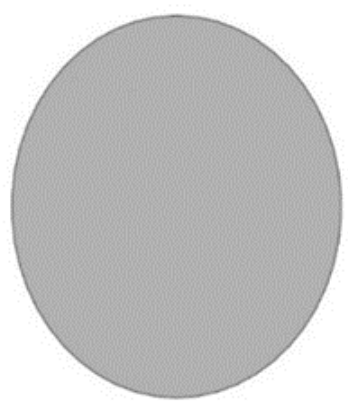

(c)

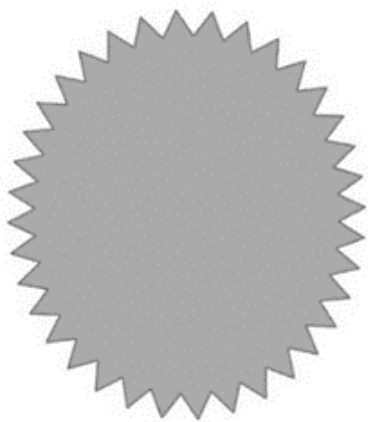

(d)

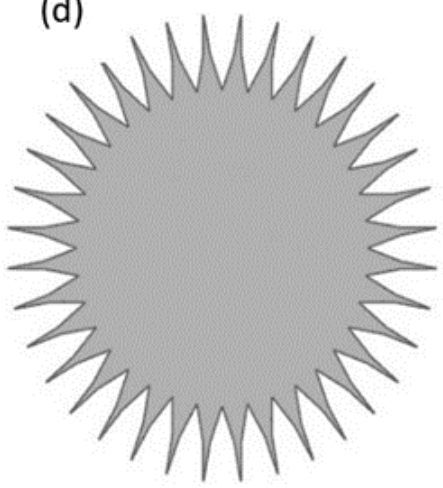

Figure 2-1 Different plate shapes: (a) reference, (b) oval (c) star Shape with $60^{\circ}$ edge (d) star shape with power-law edge. The reference consists of five rectangles, with the large one representing the main impact zone. The four small rectangles are fixation points. The oval plate is a second reference which has, like (c) and (d), an oval main impact zone, but no spikes. 
The plates were tested with a $9 \mathrm{~mm} 7.5 \mathrm{~g}$ Full Metal Jacket (FMJ) projectile with a velocity of $380 \mathrm{~m} / \mathrm{s}$. These projectiles were chosen because of the low occurrence of backscattering. Another reason is that they are NATO-compliant and widely available for such tests.

The velocity was measured $15 \mathrm{~m}$ before impact, using a BMC 31 (Kurzzeit, Germany) ballistic data processing system and an LS 260 (Kurzzeit, Germany) light gate for velocity measuring. The velocity drop within the light gate is considered, using the known drag coefficient of the specific FMJ projectile type. Knowing the velocity drop between measurement system and impact is very important to determine the reference impact velocity. In this case all four sensors are equidistant from the centre as shown in Figure 2-3b. This is easier for triangulation, which will be applied in future.

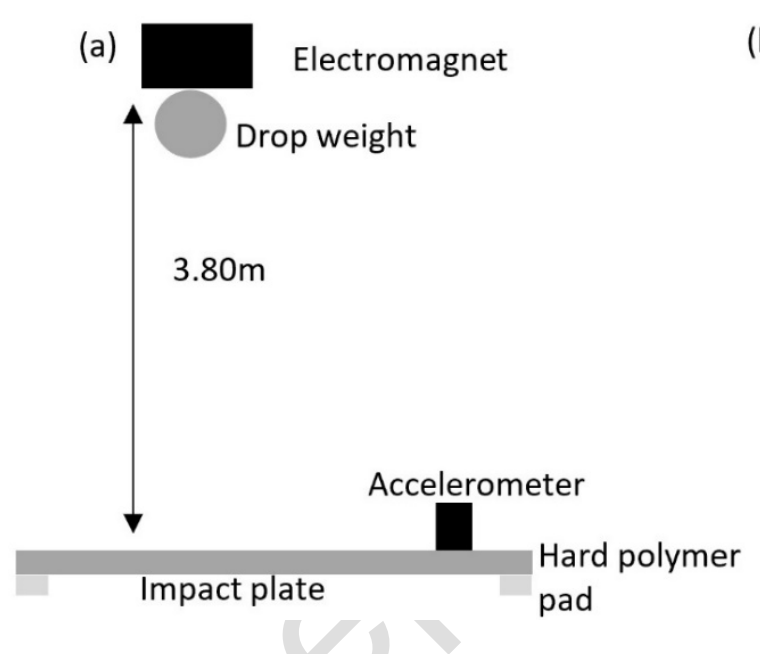

(b)

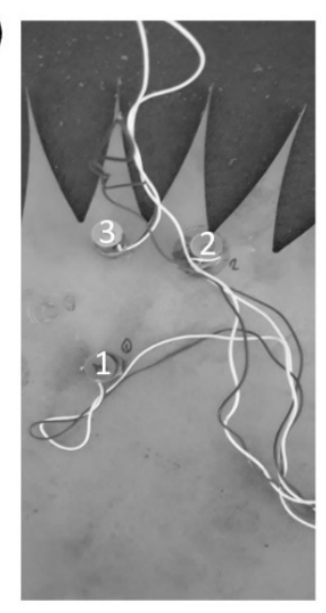

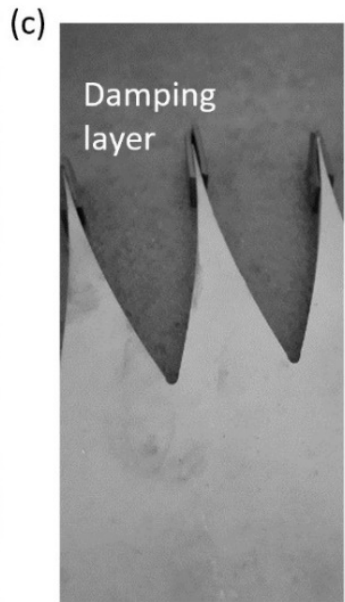

Figure 2-2: First test scenario: (a) schematic test layout, (b) accelerometer with the sensor numbers, sensor 1 is most proximal to the centre, the other two are at the same distance from the centre. Sensor $\mathbf{2}$ is exactly in front of the concave edge to see if this edge influences the signal. Sensor 3 is in line to the spike of the plate. (c) Damping layer which is basically a magnetic polymer compound which "sticks" perfectly to the contour of the plate. 
(a) Damping plate

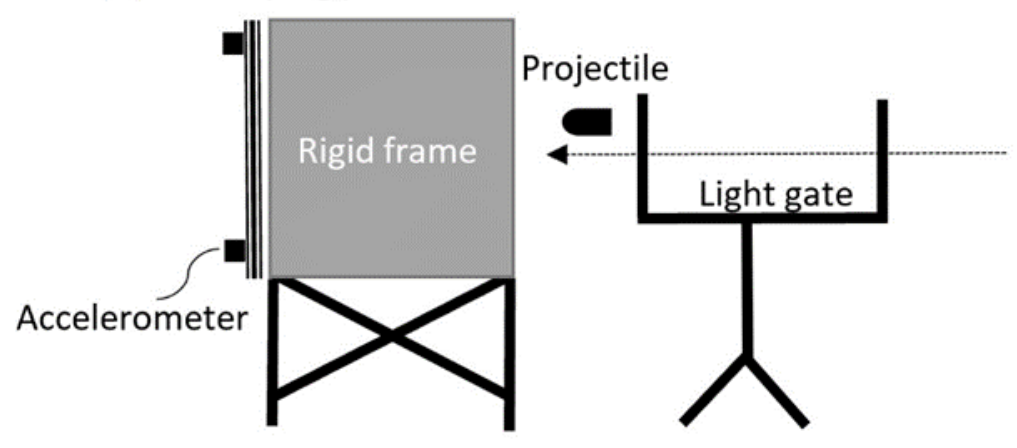

(b)

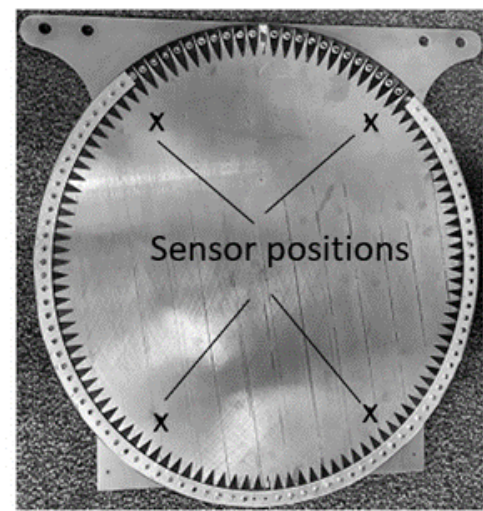

Figure 2-3: Second test scenario: (a) schematic test layout with the heavy $(800 \mathrm{~kg})$ metal frame and the plate attached to the back of the frame. (b) star-shaped plate with power-law edges with rubber damper. The plate is placed in an assembly of rubber pieces and a fixation plate suitable for mounting in the heavy metal frame.

\subsection{Results and Discussion}

\subsubsection{General Observations}

In the case of the plate with power-law edge and rubber damper, the highest excitation level occurs 10-20 $\mu$ s after impact. A similar decay pattern was observed in both experimental scenarios. This in spite of the fact that the impact energy in the second experiment was approx. 900 times higher and the plate was 30 times heavier than in the first test scenario. The results of the experiments conducted for the first and second test scenarios differ greatly in their repeatability of the impact position. In the case of the first test scenario, the radial standard deviation was less than $1 \mathrm{~mm}$. The second test scenario, performed with $9 \mathrm{~mm}$ projectiles, showed a radial standard deviation of impacts of $40 \mathrm{~mm}$. The reason for this impact distribution is the performance of the projectile acceleration system.

Interestingly, the distribution of impact positions does not affect the standard deviation between the measurements of the reverberation time shown in Table 2-1, Table 2-2. The reason for this stable reverberation time might be that, in all cases, there are some flexural waves reflected by the boundaries which keep the plate in oscillation during a few milliseconds. 
One of the most important tasks of the damped plate is to reduce the vibrations occurring after an impact event. By damping the plate, one ensures that the detected vibrations originate directly from the impact itself and not from the boundary reflections.

Figure 2-6 shows the acceleration signal acquired for the reference plate (shape like Figure 2-1a) and the star-shaped plate with power-law edges (shape like Figure 2-4f). In this figure, the reference plate shows a package of small flexural waves which are detected by the accelerometer $30 \mu \mathrm{s}$ before the main wave packages arrive. These packages disturb the positioning and the momentum measurement process, since it is not possible to distinguish between the small reflected flexural waves and the wave package which is emitted directly from the impact. This is especially true if the two wave packages are overlapping more than the ones represented in Figure 2-6a. With the proposed design, a very pure impact signal can be produced, as seen in Figure 2-6b. In this signal, no disturbances are visible before the main wave package arrives. This enhances the accuracy of the impact measurement significantly.

The magnitude of the peaks is rising steadily and no significant disturbance in the signal can be detected before the first small excitation occurs. In the case of the star-shaped plate with power-law edges with rubber damper, the acquired signal is significantly different. The package of small waves does not occur. In both cases the magnitude of the acceleration peak is about $20,000 \mathrm{~m} / \mathrm{s} 2$.

The best plate shape for damping single impacts is the star-shaped plate with the power-law edges with the damping layer. This shape is a combination of the shape of acoustic black holes and the star-shaped flyer plate. The damping effect of general acoustic black holes, as in the case of the power-law shapes, is strongly reduced, since acoustic black holes react sensitively to truncation imperfections, material failures, and surface roughness and reduced length of the last part of the spike [16]. However, with the use of rubber dampers, the damping effect is still significantly better than compared with the star-shape $60^{\circ}$ edge with the damping layer. This can only be explained by the fact that the acoustic black hole is an additional help for the star-shape geometry. 
In all measurements, there is a fast-oscillating signal body followed by sharp peaks. These peaks are occurring stochastically because the plate is excited in a turbulent regime. This makes the quantification of the damping system challenging, since the peaks of the signal decay faster than the noise.

The reverberation time was chosen as the most stable value to quantify the system. It does not depend on a specific frequency, which is very important for such turbulent systems. The results reported here are promising and a method can be developed to analyse more sophisticated impact detection systems.

\subsubsection{First Test Scenario}

The data presented in Figure 2-4 are the raw data representing typical plate vibrational signals for all plates tested in the first test scenario. One can see that the plate shape influences the signal decay pattern.

The damped and undamped power-law edged plate exhibits a steady signal decay, whereas the oval plate shows a more oscillating behaviour after the initial excitation. It is also recognisable that the peak amplitude occurs shortly after impact in the case of the power-law shaped structures. In the case of other structural shapes, especially the star-shaped plate with $60^{\circ}$ edge, however, slow rising amplitudes of the acceleration signal may occur due to internal compressive waves reflecting at the boundaries.

Compression waves travel through the plate at almost twice the velocity of flexural waves. The accelerometers detect in one direction only, and therefore do not detect these initial in-plane compressive waves. At the boundary, the compressive waves are reflected and partly converted to flexural waves. These reflected flexural waves are unwanted for the triangulation process, since they conflict with the arrival time of the unelected flexural wave caused directly by the impact. Such a behaviour can be seen in Figure 2-6a.

The damping layer has a significant influence, reducing or eliminating the low magnitude vibrations after $20 \mathrm{~ms}$, evidence of which is observed in Figure 2-4g. This is in keeping with the experimental investigations of Kyrlov and Winward 
[15]. The small damping layer can absorb the energy of small magnitude vibrations. However, this effect is not recognisable in the oval damped case.
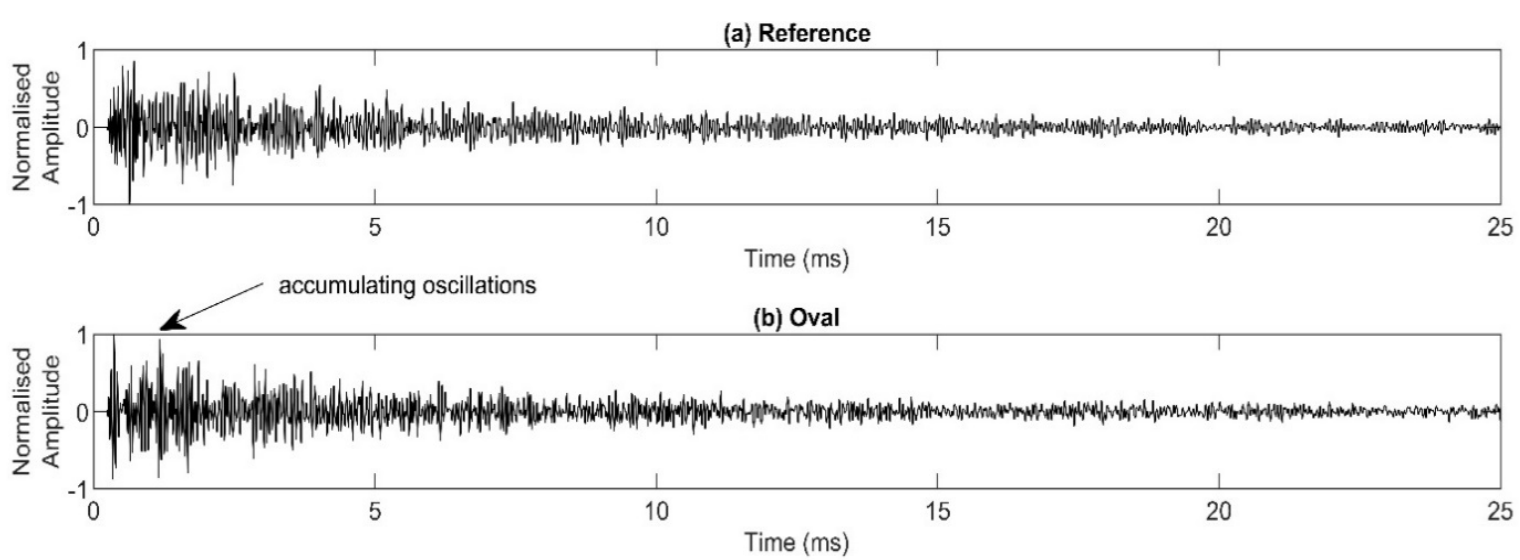

(c) Oval with damping layer
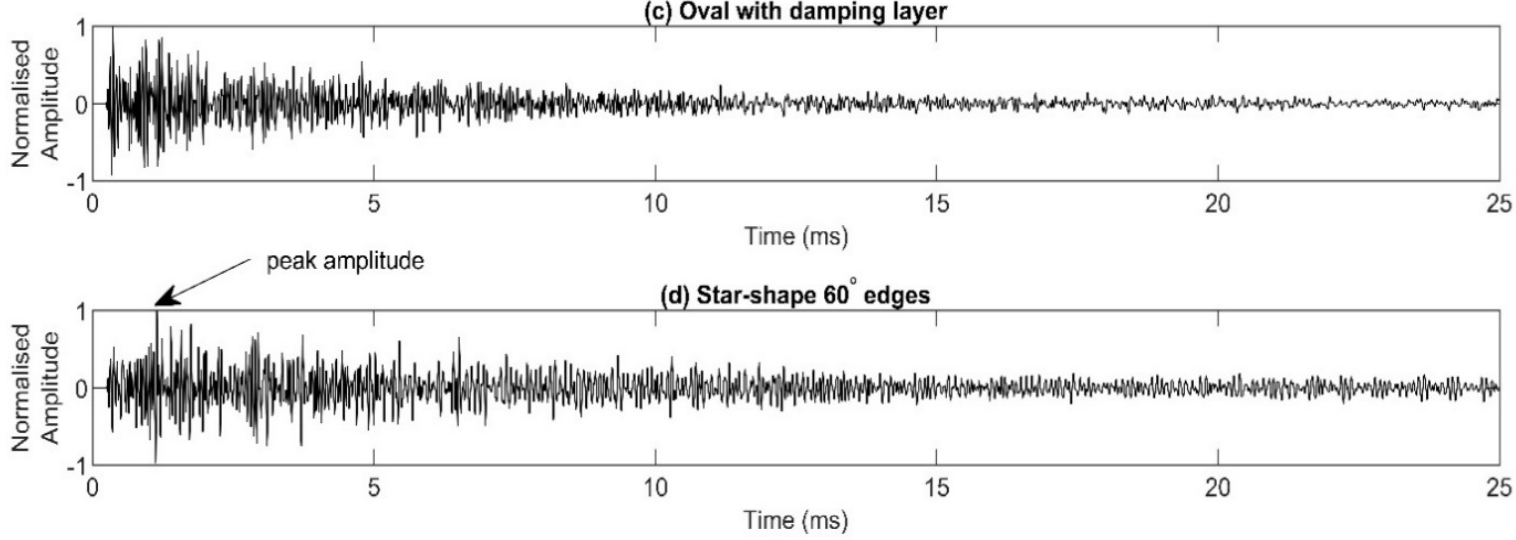

(e) Star-shape $60^{\circ}$ edges with damping layer

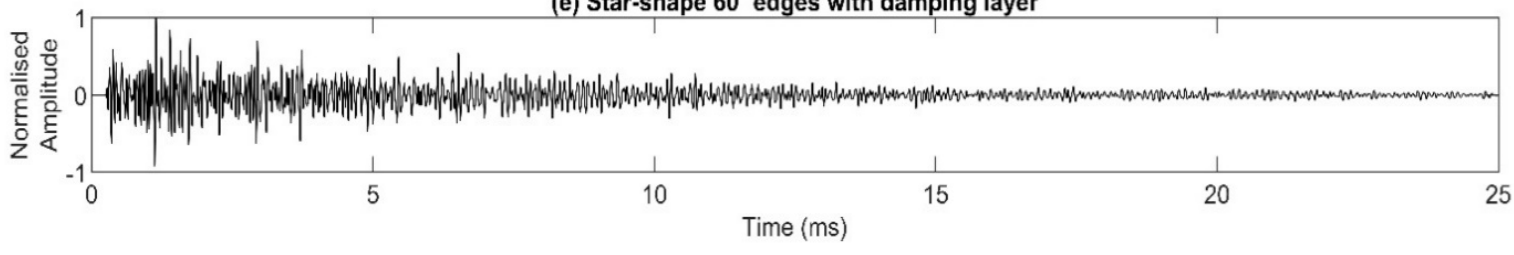

(f) Star-shape power law edges

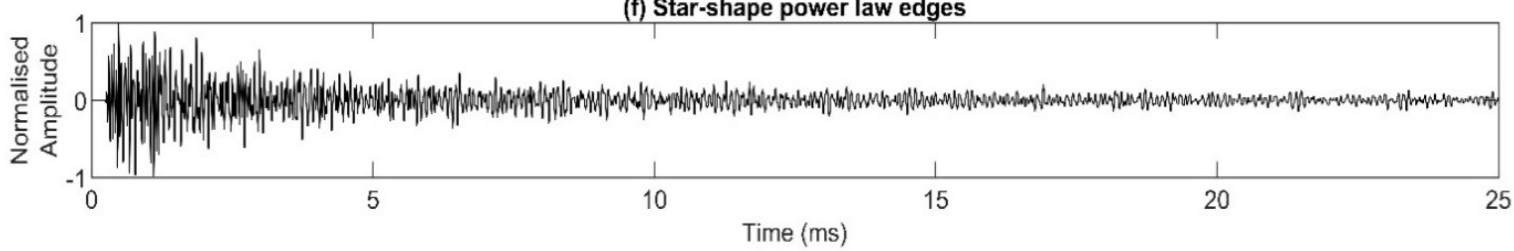

(g) Star-shape power law edges with damping layer

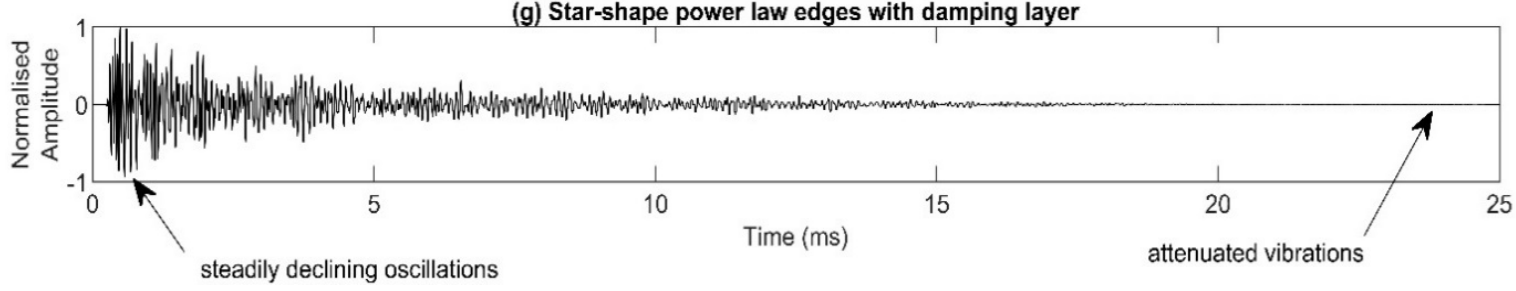

Figure 2-4a-g: Typical vibration signals of the first test 


\subsubsection{Second Test Scenario}

Figure 2-5 presents the typical data of the vibrational signal for the plates tested in the second test scenario. The decay effect of damping is easily recognisable in the case of high-velocity impacts as shown in Figure 2-5b (star-shaped plate with power-law edges with rubber damper). For the reference plate, the highest excitation level is delayed to $1 \mathrm{~ms}$ after the first oscillation is detected. The oscillations are still recognisable after $25 \mathrm{~ms}$. Comparing the reference plate with the star-shaped plate with power-law edges and rubber damper, one can see a significant reverberation time reduction from over 200 to under 10 Figure 2-6.

However, the primary aim is to improve the information/loss ratio so that the signal contains a larger portion of impact information compared with the overall information. Since the signal is strongly reduced after the first few reflections, this aim is met by using the plate with power-law shaped edge.
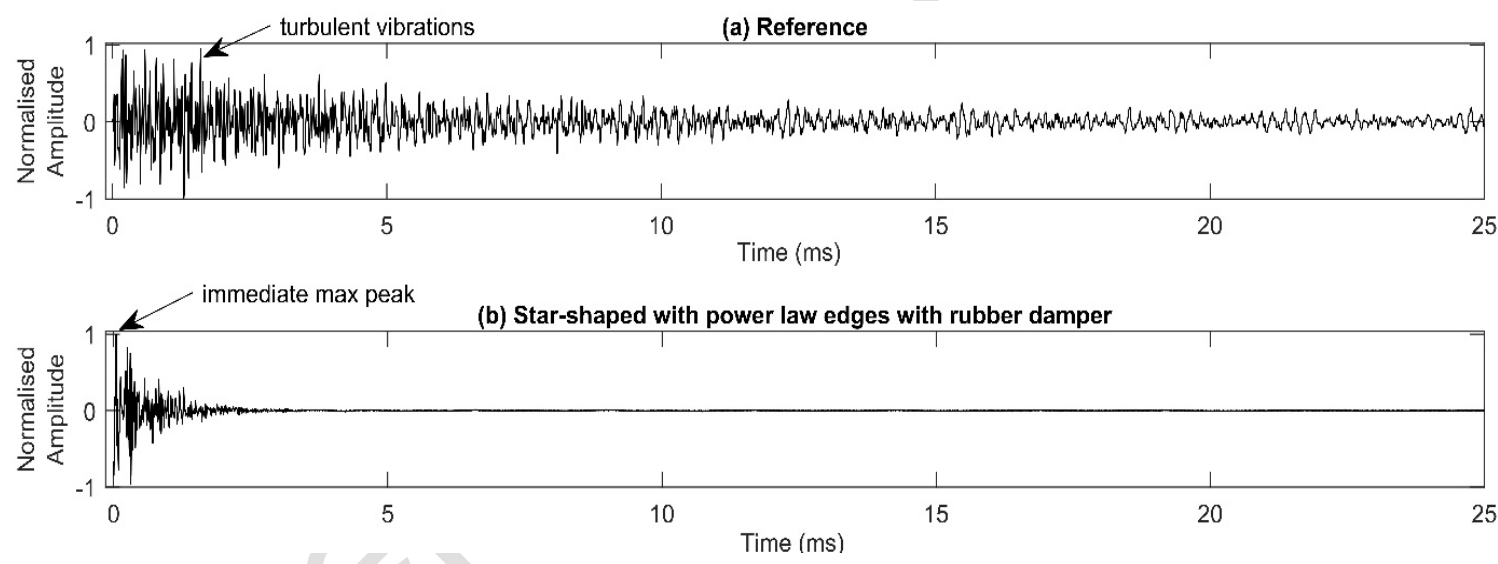

Figure 2-5: Typical vibration signals of the second test (impact of $9 \mathrm{~mm}$ projectile).

(a) The reference (rectangular) plate which shows still some oscillations after $25 \mathrm{~ms}$. (b) Shows a significant signal decay after the initial excitation, after $\mathbf{4} \mathrm{ms}$ one cannot see any remaining oscillations rising beyond the threshold. 
Table 2-1: Averages and standard deviations of 9 measurements in the first test scenario.

\begin{tabular}{|c|c|c|}
\hline Shape & $\begin{array}{l}\text { average } \\
\text { reverberation } \\
\text { time (ms) }\end{array}$ & $\begin{array}{l}\text { STD } \\
(\mathrm{ms})\end{array}$ \\
\hline Reference & 135 & 7 \\
\hline Oval & 158 & 1.2 \\
\hline Oval with damping Layer & 134 & 2.4 \\
\hline Star shape & 174 & 0.8 \\
\hline Star shape with damping layer & 63 & 0.6 \\
\hline star-shaped plate with power law edges & $147(0)$ & 1.1 \\
\hline $\begin{array}{l}\text { star-shaped plate with power law edges with } \\
\text { damping layer }\end{array}$ & 37 & 0.1 \\
\hline
\end{tabular}

Table 2-2: Averages and standard deviations of 9 measurements in the second test scenario.

\begin{tabular}{l|l|l} 
Shape & $\begin{array}{l}\text { average } \\
\text { reverberation } \\
\text { time }(\mathbf{m s})\end{array}$ & $\begin{array}{l}\text { STD } \\
\text { (ms) }\end{array}$ \\
\hline Reference & 214 & 2.9 \\
\hline $\begin{array}{l}\text { star-shaped plate with power law edges with } \\
\text { rubber damper }\end{array}$ & 9 & 0.9 \\
\hline
\end{tabular}




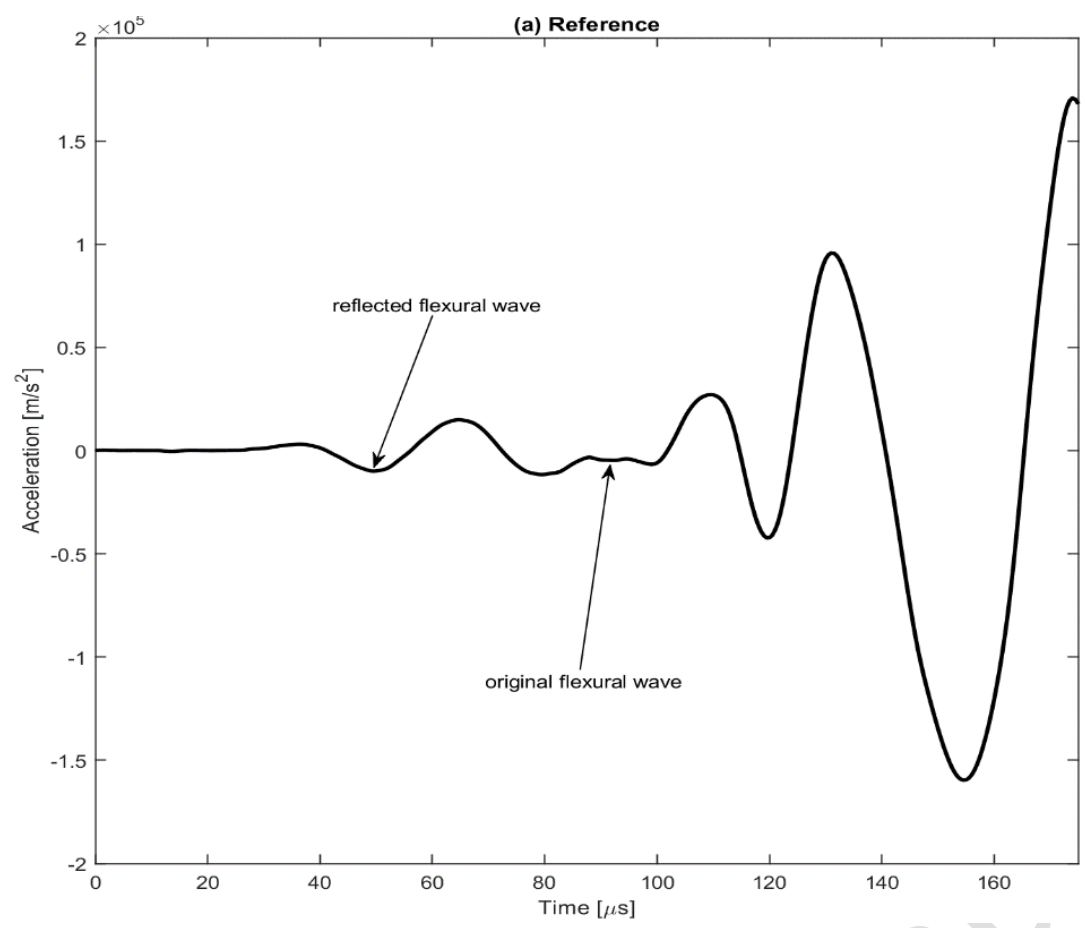

(b) Star-shaped plate with power law edges

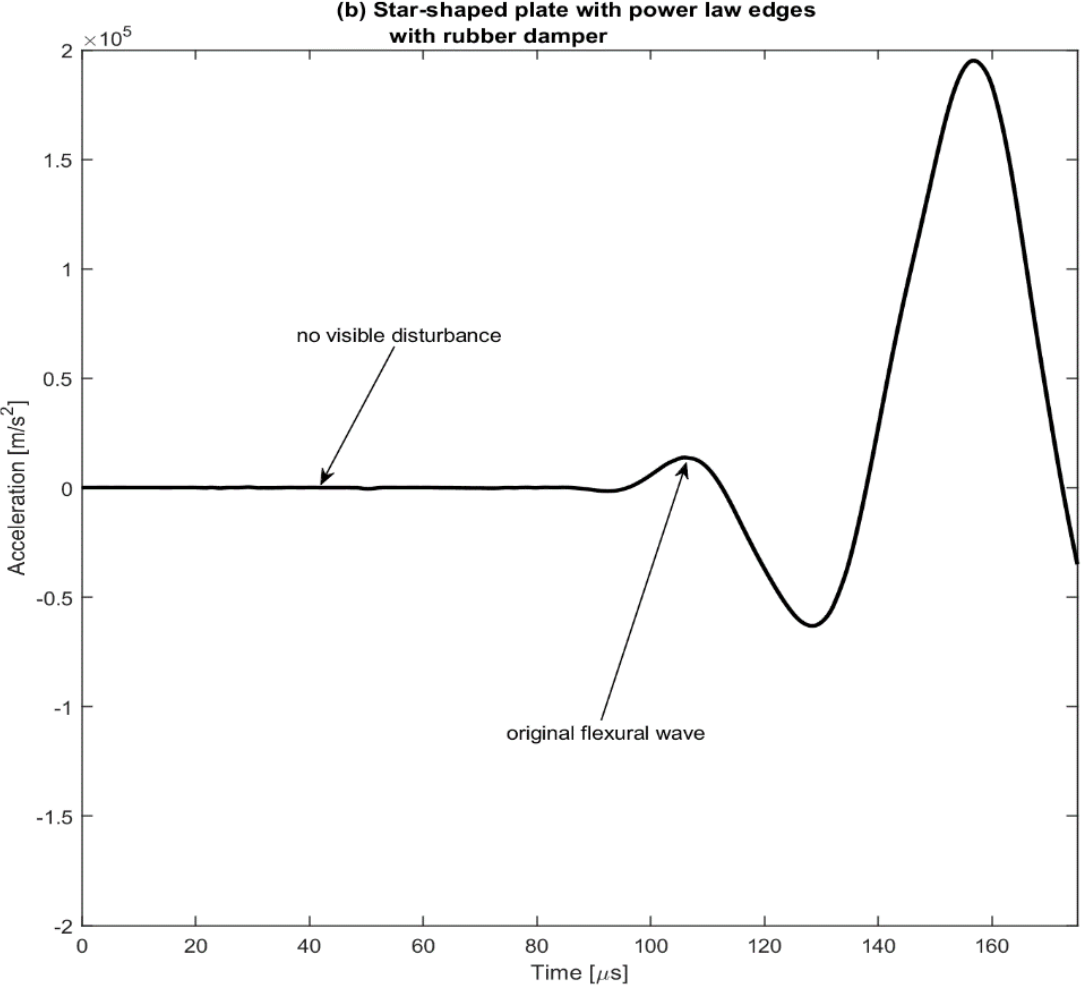

Figure 2-6: Acceleration signal acquired on (a) reference plate (b) star-shaped plate with power-law edges with rubber damper. This picture shows the benefit of the power-law edge design, the steady increase without disturbances of the wave amplitude after impact. Disturbances that can be recognised in the case of the reference plate lead to an inaccurate AT detection. 


\subsection{Conclusions}

The results show that the plate shape has a significant influence on both vibration pattern and signal decay. The reverberation time is affected by vibration damping layers in combination with toothed edges. The effect of the damped $60^{\circ}$ spike is less significant than the effect of the damped power-law shaped spike. Compared with the reference plates, it is possible to reduce the reverberation time by a factor of roughly 24 , which is a huge advantage if one uses the witness plate as measurement system.

Despite the fact that the kinetic energy from the impulse tests with a drop weight falling under gravity and a projectile flying at supersonic speed differs strongly, the oscillations resulting from vibration-time properties are comparable. The starshaped plate with power-law edges which performed best with respect to the reverberation time, can be used to design new devices which are suitable for ballistic impact investigations. For example, a ricochet measurement device capable of measuring the point of deflection on a first plate and the point of impact on a second. Furthermore, such measurement plates are also suitable for the location of multiple impacts in the same period.

It is known from the acoustic black-hole effect and from star-shaped flyer plates that they influence the wave propagation. This is particularly true with a combination of the two shapes and strong excitation bursts like ballistic impacts.

Another interesting fact is that the sound appears to be less intensive, since the acoustic reverberation time is shorter, so that it is also possible to use this system for future sound and noise reduction applications. Directly applied examples are silent bed plates for punch presses, surface metal treatments in musical situations, quieter reciprocating engines and cyclical systems. 


\subsection{References}

[1] H. D. Espinosa, "Low-Velocity Impact Testing," vol. 8, pp. 539-559, 2000.

[2] H. D. Espinosa, G. Raiser, R. J. Clifton, and M. Ortiz, "Performance of the star-shaped flyer in the study of brittle materials: Three dimensional computer simulations and experimental observations," J. Appl. Phys., vol. 72, no. 8, pp. 3451-3457, 1992.

[3] R. Morsy, H. Marzouk, M. Haddara, and X. Gu, "Multi-channel random decrement smart sensing system for concrete bridge girders damage location identification," Eng. Struct., vol. 143, pp. 469-476, 2017.

[4] U. Dahlen, N. Ryden, and A. Jakobsson, "Damage identification in concrete using impact non-linear reverberation spectroscopy," NDT E Int., vol. 75, pp. 15-25, 2015.

[5] F. Allaeys, G. Luyckx, W. Van Paepegem, and J. Degrieck, "Characterization of real and substitute birds through experimental and numerical analysis of momentum, average impact force and residual energy in bird strike on three rigid targets: a flat plate, a wedge and a splitter," Int. J. Impact Eng., 2016.

[6] C. I. Hammetter, R. L. Jones, H. L. Stauffacher, and T. F. Schoenherr, "Measurement and modeling of supersonic hailstone impacts," Int. J. Impact Eng., vol. 99, pp. 48-57, 2017.

[7] A. Agnello, J. Dosch, R. Metz, R. Sill, and P. Walter, "Acceleration Sensing Technologies for Severe Mechanical Shock," Sound Vib., vol. 48, no. 2, pp. 8U20, 2014.

[8] T. H. and O. C. and G. D. and C. J. and S. R. and C. Touzé, "Wave turbulence in vibrating plates: The effect of damping," EPL (Europhysics Lett., vol. 102, no. 3, p. 30002, 2013. 
[9] G. Zhao, H. Hu, S. Li, L. Liu, and K. Li, "Localization of impact on composite plates based on integrated wavelet transform and hybrid minimization algorithm," Compos. Struct., 2017.

[10] A. Perelli, L. De Marchi, A. Marzani, and N. Speciale, "Frequency warped cross-wavelet multiresolution analysis of guided waves for impact localization," Signal Processing, vol. 96, no. PART A, pp. 51-62, 2014.

[11] D. Mušicki, R. Kaune, and W. Koch, "Mobile emitter geolocation and tracking using TDOA and FDOA measurements," IEEE Trans. Signal Process., vol. 58, no. 3 PART 2, pp. 1863-1874, 2010.

[12] F. Gustafsson and F. Gunnarsson, "Positioning using time-difference of arrival measurements," in IEEE International Conference on Acoustics, Speech, and Signal Processing, 2003, vol. 6, p. VI-553-6 vol.6.

[13] M. Liu, J. Yang, Y. Cao, W. Fu, and Y. Cao, "A new method for arrival time determination of impact signal based on HHT and AIC," Mech. Syst. Signal Process., vol. 86, no. October 2016, pp. 177-187, 2017.

[14] V. V. Krylov and F. J. B. S. Tilman, "Acoustic 'black holes' for flexural waves as effective vibration dampers," J. Sound Vib., vol. 274, no. 3-5, pp. 605619, 2004.

[15] V. V. Krylov and R. E. T. B. Winward, "Experimental investigation of the acoustic black hole effect for flexural waves in tapered plates," J. Sound Vib., vol. 300, no. 1-2, pp. 43-49, 2007.

[16] E. Bowyer and V. Krylov, "Acoustic black hole manufacturing for practical applications and the effect of geometrical and material imperfections," no. 4, pp. 2411-2421, 2016.

[17] J. Y. Lee and W. Jeon, "Vibration damping using a spiral acoustic black hole," J. Acoust. Soc. Am., vol. 141, no. 3, pp. 1437-1445, 2017.

[18] A. Howard, "Morphological control of censile release in ceramic penetration," Cranfield University, 2014. 
[19] Z." Nakutis and P. Kaškonas, "Bridge vibration logarithmic decrement estimation at the presence of amplitude beat," Meas. J. Int. Meas. Confed., vol. 44, no. 2, pp. 487-492, 2011.

[20] M. Feldman and S. Braun, "Nonlinear vibrating system identification via Hilbert decomposition," Mech. Syst. Signal Process., vol. 84, pp. 65-96, 2017.

[21] Y. Liao and V. Wells, "Modal parameter identification using the log decrement method and band-pass filters," J. Sound Vib., vol. 330, no. 21, pp. 5014-5023, 2011.

[22] J. Lardies and S. Gouttebroze, "Identification of modal parameters using the wavelet transform," Int. J. Mech. Sci., vol. 44, no. 11, pp. 2263-2283, 2002.

[23] P. Avanesians and M. Momayez, "Wave separation: Application for arrival time detection in ultrasonic signals," Ultrasonics, vol. 55, no. 1, pp. 15-25, 2015.

[24] M. R. Schroeder, "New method of measuring reverberation time," Journal of the Acoustical Society of America, vol. 37. pp. 409-412, 1965.

[25] K. Arcas and A. Chaigne, "On the quality of plate reverberation," Appl. Acoust., vol. 71, no. 2, pp. 147-156, 2010.

[26] V. Gomez Escobar and J. M. Barrigon Morillas, "Analysis of intelligibility and reverberation time recommendations in educational rooms," Appl. Acoust., vol. 96, pp. 1-10, 2015.

[27] R. R. Boullosa, "Vibration measurements in the classical guitar," Appl. Acoust., vol. 63, no. 3, pp. 311-322, 2002.

[28] W. Dudziński, Ł. Konat, and G. Pękalski, "Structural and strength characteristics of wear-resistant martensitic steels," Arch. Foundry Eng., vol. 8, no. 2, pp. 21-26, 2008. 